\title{
Anticoagulant Use, the Prevalence of Bridging, and Relation to Length of Stay among Hospitalized Patients with Non-Valvular Atrial Fibrillation
} \author{
and Alpesh Amin ${ }^{3}$ \\ 1 Truven Health Analytics Inc., Washington, DC, USA \\ 2 Boehringer Ingelheim Pharmaceuticals, Inc., Ridgefield, CT, USA \\ 3 University of California, Irvine, CA, USA
}

Karen Smoyer-Tomic, ${ }_{1}^{1}$ Kimberly Siu, ${ }^{2}$ David R. Walker, ${ }^{2}$ Barbara H. Johnson, ${ }^{1}$ David M. Smith, ${ }^{1}$ Stephen Sander ${ }^{2}$

\begin{abstract}
Objective: The objectives of this study were to describe inpatient anticoagulation and bridging in patients with non-valvular atrial fibrillation (NVAF) and to identify whether differences exist in length of stay (LOS) among bridged versus non-bridged NVAF patients.

Design: Administrative claims data were used to select patients $\geq 18$ years with a primary or secondary discharge diagnosis of NVAF and inpatient warfarin use from 1 July 2004 to 30 September 2009. Patients with valvular or transient causes of NVAF or pregnancy were excluded. Inpatient bridging was defined as receipt of an anticoagulant in addition to warfarin during the hospitalization. LOS was reported for nonbridged patients (warfarin only) and compared with three bridging regimens: low molecular weight heparin/ pentasaccharide (LMWH/PS); unfractionated heparin (UFH); and two-agent bridging (LMWH/PS and UFH). Multivariate analyses were performed to evaluate the association between bridging and LOS, adjusting for demographic and clinical variables.

Results: Of 6340 NVAF patients, $48 \%$ received inpatient warfarin (mean LOS 5.5 days); among them, $64 \%$ received bridging therapy (mean LOS 6.3 days) [LMWH/PS 45\% (mean LOS 5.6 days), UFH 36\% (mean LOS 6.0 days), two-agent bridging $18 \%$ (mean LOS 8.4 days)]. Following multivariate analysis, relative to patients who received inpatient warfarin only, LOS was significantly higher for patients with UFH (19.3\%) and patients with two-agent bridging (45.1\%). Patients with pre-period warfarin, cancer, or diabetes mellitus who received bridging agents had significantly longer LOS than patients with those conditions who were not bridged. Conclusion: LOS was longer for bridged than non-bridged patients. Further studies are needed to identify predictors of bridging and to explain why bridged NVAF patients had longer LOS.
\end{abstract}

\section{Introduction}

Atrial fibrillation (AF) is a frequently occurring cardiac arrhythmia and a significant risk factor for ischemic stroke. ${ }^{[1-4]}$ Prevalence in the US is estimated at 3 million persons and increases with age, occurring in approximately $9 \%$ of the population aged $80-89$ years. ${ }^{[5,6]}$ Over a third of AF patients are hospitalized each year, with hospitalization costs accounting for \$US2.3 billion of the \$US6 billion annual incremental AFspecific costs. ${ }^{[1]}$ Anticoagulation (AC) has proven effective in the prevention of stroke in patients with $\mathrm{AF},{ }^{[7-10]}$ yet approximately $55 \%$ of patients are untreated, resulting in total direct costs from stroke to Medicare of \$US4.8 billion. ${ }^{[6]}$ Guidelines from the American Heart Association/American Stroke Association (AHA/ASA) for the primary prevention of stroke recommend use of warfarin in non-valvular AF (NVAF) patients at high risk for stroke and also for patients at moderate risk if they can be safely treated. ${ }^{[9]}$ There are occasions when patients require AC but when warfarin, due to its relatively slow onset and long halflife, may need to be temporarily discontinued, delayed, or supplemented with a faster onset parenteral AC agent until it takes effect. In these cases, bridging therapy may be used.

Bridging therapy is the transition of one AC agent to another and has two major applications. Bridging therapy most commonly 
describes peri-procedural AC in patients maintained on warfarin therapy but who need to transition from warfarin to a parenteral AC agent (typically low molecular weight heparin $[\mathrm{LMWH}]$ or unfractionated heparin $[\mathrm{UFH}])$ to maintain thromboprophylaxis while reducing the risk of bleeding during surgery. ${ }^{[1-13]}$ The second bridging therapy application is acute $\mathrm{AC}$ with a rapid onset, parenteral $\mathrm{AC}$ as a short-term transition to either de novo warfarin therapy or when resuming interrupted long-term warfarin therapy. ${ }^{[14,15]}$ Transition phases can include the time period around the initiation of warfarin, when it may be necessary to quickly achieve a therapeutic international normalized ratio (INR) in patients at risk for embolic events, and the time period surrounding planned or unplanned surgical or other invasive procedures, when it may be necessary to interrupt existing warfarin therapy to reduce bleeding risk. AHA/ASA guidelines on prevention of stroke in AF patients with stroke or transient ischemic attack (TIA) consider use of LMWH as bridging therapy 'reasonable' to fill the gap in AC in high-risk AF patients who must interrupt AC therapy. ${ }^{[10]}$ The American College of Chest Physicians (ACCP) 2008 practice guidelines suggest that $\mathrm{AF}$ patients at high or moderate risk for thromboembolism receive therapeutic dose subcutaneous (SC) LWMH (preferred) or intravenous (IV) UFH as bridging therapy in patients requiring temporary peri-operative interruption of warfarin. ${ }^{[11]}$ Despite these guidelines, there is a lack of consensus among clinicians on an appropriate bridging therapy strategy, which tends to be based on the clinical judgment as well as personal opinion of the attending physician. ${ }^{[13]}$ Available data do not adequately address the benefits and risks of bridging therapy, and questions remain on indications for its use and the choice, dosing, and timing of bridging agents. ${ }^{[16-18]}$

The objectives of the current study are to describe AC treatment in the inpatient setting in patients hospitalized with NVAF, to determine the prevalence of inpatient bridging therapy, and to identify whether differences exist in length of stay (LOS) among bridged versus non-bridged NVAF patients, after controlling for demographic and clinical differences between the two groups. In this study, a broad definition of bridging was used, identified by evidence of receipt in the inpatient setting of both warfarin and a parenteral agent, without regard for timing of bridging agents or bridging indication.

\section{Design}

\section{Data Sources}

Three Truven Health Analytics MarketScan ${ }^{\circledR}$ databases were used in this study: the Hospital Drug Database (HDD), the
Commercial Claims and Encounters (Commercial) Database, and the Medicare Supplemental and Coordination of Benefits (Medicare) Database. The HDD is derived primarily from the hospital billing systems of 584 hospitals. Data elements include admission and discharge dates, primary and secondary diagnoses and procedures, patient demographics, drug utilization, and supplies.

The Commercial database contains the healthcare experience of privately insured individuals covered under a variety of fee-for-service, fully capitated, and partially capitated health plans. The database is constructed from claims and enrollment data provided by large employer-sponsored health plans from across the USA. Approximately 35 million individuals are included annually in the database, encompassing employees, their spouses, and dependents. The Medicare database contains the healthcare experience of approximately 2.5 million individuals annually with Medicare Supplemental insurance paid for by employers.

The Commercial and Medicare data include medical claims for healthcare services performed in both the inpatient and outpatient settings and enrollment data including member demographic information and eligibility and benefits data. The medical claims files include service dates, provider reimbursement amounts, and patient co-payment and deductible amounts. Within the Medicare database, both the Medicare-covered portion of payment (represented as the coordination of benefits amount) and the employer-paid portion are included, as well as any out-of-pocket patient expenses. The medical care claims are linked to complete outpatient prescription drug claims, including claims for mail order prescriptions and specialty pharmacy, which fully capture prescription fills from all locations. These databases include persons from all US States and are de-identified and fully compliant with the Health Insurance Portability and Accountability Act of 1996. MarketScan data quality has been assessed and the comprehensiveness of the data has been confirmed through frequent audits of the data. There are more than 400 publications using MarketScan data.

Because this study did not involve the collection, use, or transmittal of individually identifiable data, Institutional Review Board review or approval was not required.

\section{Patient Selection}

Patients $\geq 18$ years of age with a primary or secondary discharge diagnosis of AF [International Classification of Diseases, 9th Edition, Clinical Modification (ICD-9-CM) diagnosis code 427.31] from 1 July 2004 through 30 September 2009 (enrollment window) were selected from the HDD. The subset of HDD AF patients also included in the Commercial and Medicare 
databases was identified. Patients were required to have a minimum of 6 months of continuous medical and pharmacy coverage prior to the admission date of the first AF admission (index admission) in the enrollment window and a minimum of 30 days of coverage subsequent to the discharge date of the index admission, as well as complete data for all study variables. For patients with multiple AF admissions, only the index admission was evaluated. Index admissions were required to have both admission and discharge dates. Since there is no diagnosis for NVAF, patients were excluded if claims for a valvular cause or transient cause of AF were present during the index admission, specifically pulmonary embolism, cardiac surgery, or hyperthyroidism, to limit AF admissions to those most representative of patients with NVAF. Patients with evidence of hyperthyroidism in the 6-month pre-index period were also excluded, as were patients with pregnancy at any time in the study window, defined as the 6-month pre-index period through the 30-day post-index period. All study patients were required to have evidence of warfarin use during the index admission to be included in the bridging analysis.

\section{Outcomes}

The number and percentage of NVAF patients with anticoagulant use during the index admission were quantified, overall and by specific agent. AC was reported for the entire NVAF group, and also stratified by presence/absence of inpatient bridging therapy. Bridging therapy was defined as receipt of a second anticoagulant (bridging agent) in addition to warfarin during the index admission. Three bridging agent regimens were evaluated: LMWH or other pentasaccharide (e.g. fondaparinux) [LMWH/PS]; unfractionated heparin (UFH) [excluding UFH used for line flushing]; and two-agent bridging (LMWH/PS and UFH).

LOS for the index admission was measured and reported for patients on warfarin alone and for patients with inpatient bridging therapy by bridging agent.

\section{Variables}

Demographic, utilization, and cost variables measured in the 6 months prior to the index admission and reported by bridging therapy status included mean age, age group, sex, payer type (commercial, Medicare), plan type (fee-for-service, managed care, other/unknown), population density (urban, rural, unknown), geographic region (Northeast, North Central, South, West, unknown), concomitant medications of interest (including warfarin), previous hospitalization, and mean and median total pre-period healthcare payments (inflation adjusted to 2009 dollars based on the Medical Care Consumer Price Index). Specific co-morbid conditions were measured in the 6-month pre-period as well as during the index admission. The Charlson Comorbidity Index (Deyo version) $[\mathrm{CCI}],{ }^{[19]}$ based on the presence or absence of specific ICD-9-CM diagnosis codes consistent with 19 conditions, was also measured, as was the $\mathrm{CHA}_{2} \mathrm{DS}_{2}$-VASc score, a predictor of the clinical risk of stroke and thromboembolism in patients with AF. ${ }^{[20]}$

\section{Statistical Analyses}

Descriptive statistics were reported by inpatient bridged/ non-bridged categories for demographic and clinical variables in the pre-hospitalization period as well as by inpatient medications for patients receiving inpatient warfarin. Because LOS is a skewed variable, multivariate analysis was performed using a generalized linear model (GLM) with a log link and gamma error distribution to evaluate the association between bridging therapy and LOS, adjusting for demographic and clinical variables. All model fitting was done using PROC GENMOD in SAS version 9.2 (SAS, Cary, NC, USA).

For ease of understanding the modeled relation between study variables and LOS, results were presented as 'percentage change' relative to a reference LOS for a 'low risk' NVAF patient, as well as total days hospitalized during the index admission. The reference patient was defined as a 73 -year-old male who received inpatient warfarin without a bridge agent, not from the Western region, with total 6-month pre-period costs of \$US8800 and all other variables equal to 'No.' In addition to calculating the parameter estimates and confidence intervals, PROC GENMOD also produced the predicted LOS associated with a change in one variable (e.g. 'no' to 'yes'), for a patient with one particular characteristic and all other factors the same as the reference. To estimate the percent change in LOS and days LOS for patients with both a bridge agent and an additional condition (e.g. cancer and LWMH/PS bridging), the calculations were more complex and included contributions to LOS identified by the model for both main effects and the interaction effect. The interactions include the extra effects, either synergistic or antagonistic, that occur when both factors are present, whether over and above (synergistic) or under and below (antagonistic) those of the two factors being present on their own.

The models were used to compare estimated LOS in the low risk NVAF reference group (e.g. none of the pre-selected comorbid conditions) that did not receive bridging therapy to three types of NVAF patients: (i) those who were also low risk but received inpatient bridging therapy; (ii) those with selected 
conditions (e.g. cancer or diabetes mellitus) who did not receive bridging therapy; and (iii) those with a selected co-morbidity who received bridging therapy. This method controlled for the demographic and clinical variables described above and reported in tables I and II.

Table I. Demographic characteristics of NVAF patients by bridging therapy

\begin{tabular}{|c|c|c|c|c|c|}
\hline \multirow[t]{3}{*}{ Characteristic } & \multicolumn{4}{|c|}{ NVAF patients on warfarin } & \multirow[t]{3}{*}{$p$-Value } \\
\hline & \multicolumn{2}{|c|}{$\begin{array}{l}\text { Bridged }^{\mathrm{a}} \\
(\mathrm{N}=1944)\end{array}$} & \multicolumn{2}{|c|}{$\begin{array}{l}\text { Non-bridged }^{b} \\
(\mathrm{~N}=1093)\end{array}$} & \\
\hline & $\mathrm{n} /$ mean & $\% / S D$ & $\mathrm{n} /$ mean & $\% / S D$ & \\
\hline Age (years) & 72.3 & 10.8 & 75.3 & 9.8 & $<0.001$ \\
\hline Age group (years) & & & & & $<0.001$ \\
\hline $18-24$ & 1 & $0.1 \%$ & 0 & $0.0 \%$ & \\
\hline $25-34$ & 3 & $0.2 \%$ & 0 & $0.0 \%$ & \\
\hline $35-44$ & 23 & $1.2 \%$ & 4 & $0.4 \%$ & \\
\hline $45-54$ & 102 & $5.2 \%$ & 35 & $3.2 \%$ & \\
\hline $55-64$ & 304 & $15.6 \%$ & 116 & $10.6 \%$ & \\
\hline $65+$ & 1511 & $77.7 \%$ & 938 & $85.8 \%$ & \\
\hline Sex & & & & & 0.426 \\
\hline Male & 1098 & $56.5 \%$ & 601 & $55.0 \%$ & \\
\hline Female & 846 & $43.5 \%$ & 492 & $45.0 \%$ & \\
\hline Payer & & & & & $<0.001$ \\
\hline Commercial & 426 & $21.9 \%$ & 152 & $13.9 \%$ & \\
\hline Medicare & 1518 & $78.1 \%$ & 941 & $86.1 \%$ & \\
\hline Insurance plan type & & & & & $<0.001$ \\
\hline Fee-for-service & 905 & $46.6 \%$ & 585 & $53.5 \%$ & \\
\hline Managed care & 997 & $51.3 \%$ & 495 & $45.3 \%$ & \\
\hline Other/unknown & 42 & $2.2 \%$ & 13 & $1.2 \%$ & \\
\hline Population density & & & & & 0.719 \\
\hline Urban & 1606 & $82.6 \%$ & 909 & $83.2 \%$ & \\
\hline Rural & 334 & $17.2 \%$ & 183 & $16.7 \%$ & \\
\hline Unknown & 4 & $0.2 \%$ & 1 & $0.1 \%$ & \\
\hline Geographic region & & & & & 0.569 \\
\hline Northeast & 40 & $2.1 \%$ & 15 & $1.4 \%$ & \\
\hline North central & 500 & $25.7 \%$ & 296 & $27.1 \%$ & \\
\hline South & 1364 & $70.2 \%$ & 762 & $69.7 \%$ & \\
\hline West & 36 & $1.9 \%$ & 19 & $1.7 \%$ & \\
\hline Unknown & 4 & $0.2 \%$ & 1 & $0.1 \%$ & \\
\hline
\end{tabular}

a Bridged = inpatient warfarin with inpatient LMWH/PS and/or with UFH.

b Non-bridged = inpatient warfarin without inpatient LMWH/PS or UFH.

LMWH/PS = low molecular weight heparin/pentasaccharide; $\mathbf{N}=$ number of patients in database sample meeting study selection criteria; $\mathbf{n}=$ number of patients in database sample meeting study selection criteria with specified demographic characteristic; NVAF = non-valvular atrial fibrillation; UFH= unfractionated heparin.

\section{Results}

Non-Valvular Atrial Fibrillation (NVAF) Patients, Inpatient Anticoagulant Use, and Length of Stay

A total of 6340 patients had a hospitalization for NVAF from 1 July 2004 through 30 September 2009 and met the selection criteria for age, eligibility, and exclusionary diagnoses (figure 1). Seventy percent of NVAF hospitalizations were medical admissions; $30 \%$ were surgical admissions.

Of the $6340 \mathrm{NVAF}$ patients, $76 \%$ received inpatient warfarin, LMWH/PS, or UFH (figure 2). Another $9 \%$ received an antiplatelet or an AC other than warfarin, LMWH/PS, or UFH. Fifteen percent of NVAF patients received no AC. Among the $48 \%(\mathrm{n}=3037)$ of patients who received inpatient warfarin, $64 \%$ $(\mathrm{n}=1944)$ received bridging therapy. LMWH/PS accounted for $45 \%$ of bridging therapy, UFH for $36 \%$, and two-agent bridging therapy for $18 \%$. The descriptive tables and multivariate analyses were confined to the 3037 patients that received inpatient warfarin, stratified by receipt of inpatient bridging therapy versus inpatient warfarin alone.

The mean LOS for the 6340 NVAF patients was 5.2 days (SD 5.5 days); median LOS was 4 days. Of the 3037 NVAF patients receiving inpatient warfarin (alone or in combination with a bridging agent), mean and median LOS were 5.5 days (SD 5.2 days) and 4 days. Among NVAF patients not receiving inpatient warfarin, mean LOS was 5.0 days (SD 5.7 days), with a median LOS of 3 days.

\section{NVAF Patients: Bridged versus Non-Bridged}

Bridged patients were younger (table I), and were more likely to be covered under a commercial payer and to have a managed care health plan than non-bridged patients receiving warfarin alone. No differences by sex, urban/rural location, or region were observed (table I). Bridged patients also were slightly healthier, as indicated by lower $\mathrm{CCI}, \mathrm{CHA}_{2} \mathrm{DS}_{2}$-VASc [(i) Congestive heart failure or left ventricular dysfunction, 1 pt, (ii) Hypertension, $1 \mathrm{pt}$, (iii) Age $\geq 75$ years, 2 pts, (iv) Diabetes mellitus, 1 pt, (v) Stroke, transient ischemic attack, or thromboembolism, 2 pts, (vi) Vascular disease (prior myocardial infarction, peripheral artery disease, aortic plaque), 1 pt, (vii) $65 \leq \mathrm{Age}<75,1 \mathrm{pt}$, (viii) Sex category, $1 \mathrm{pt}$ if female)] score, and pre-period inpatient utilization and healthcare costs, than non-bridged patients (table II). There were some differences in co-morbid conditions assessed in the preperiod, with higher rates of acute myocardial infarction (AMI), atrial flutter, and ischemic stroke/TIA/cerebrovascular disease in bridged patients than non-bridged patients. 
Table II. Clinical characteristics of NVAF patients in the 6-month pre-period and during the index hospitalization

\begin{tabular}{|c|c|c|c|c|c|}
\hline \multirow[t]{3}{*}{ Characteristic } & \multicolumn{4}{|c|}{ NVAF patients on warfarin } & \multirow[t]{3}{*}{$p$-Value } \\
\hline & \multicolumn{2}{|c|}{ Bridged $^{a}(N=1944)$} & \multicolumn{2}{|c|}{ Non-bridged $^{\mathrm{b}}(\mathrm{N}=1093)$} & \\
\hline & $\bar{n} /$ mean & $\% / S D$ & $\overline{\mathrm{n} / \text { mean }}$ & $\% / S D$ & \\
\hline \multicolumn{6}{|l|}{ Co-morbidity/risk indicators } \\
\hline Charlson-Deyo Comorbidity Index & 1.1 & 1.5 & 1.3 & 1.6 & $<0.001$ \\
\hline $\mathrm{CHA}_{2} \mathrm{DS}_{2}$-VASc Score & 3.5 & 1.7 & 3.8 & 1.5 & $<0.001$ \\
\hline \multicolumn{6}{|l|}{ Co-morbid conditions } \\
\hline Cancer & 171 & $8.8 \%$ & 81 & $7.4 \%$ & 0.184 \\
\hline Rheumatoid arthritis & 37 & $1.9 \%$ & 22 & $2.0 \%$ & 0.834 \\
\hline Multiple sclerosis & 1 & $0.1 \%$ & 3 & $0.3 \%$ & 0.104 \\
\hline Coronary artery disease & 793 & $40.8 \%$ & 493 & $45.1 \%$ & 0.021 \\
\hline Acute myocardial infarction & 73 & $3.8 \%$ & 20 & $1.8 \%$ & 0.003 \\
\hline Cardiomyopathy & 200 & $10.3 \%$ & 122 & $11.2 \%$ & 0.453 \\
\hline Ischemic stroke/TIA/cerebrovascular disease & 175 & $9.0 \%$ & 73 & $6.7 \%$ & 0.025 \\
\hline Heart failure & 555 & $28.5 \%$ & 391 & $35.8 \%$ & $<0.001$ \\
\hline Atrial flutter & 225 & $11.6 \%$ & 69 & $6.3 \%$ & $<0.001$ \\
\hline Hypertension & 1396 & $71.8 \%$ & 787 & $72.0 \%$ & 0.91 \\
\hline Peripheral artery disease & 193 & $9.9 \%$ & 95 & $8.7 \%$ & 0.264 \\
\hline Liver disease & 28 & $1.4 \%$ & 21 & $1.9 \%$ & 0.313 \\
\hline Renal disease & 379 & $19.5 \%$ & 225 & $20.6 \%$ & 0.47 \\
\hline COPD/emphysema & 404 & $20.8 \%$ & 238 & $21.8 \%$ & 0.52 \\
\hline Hypothyroidism & 285 & $14.7 \%$ & 183 & $16.7 \%$ & 0.127 \\
\hline Diabetes mellitus & 552 & $28.4 \%$ & 304 & $27.8 \%$ & 0.732 \\
\hline Peptic ulcer/GERD & 305 & $15.7 \%$ & 152 & $13.9 \%$ & 0.187 \\
\hline VTE & 71 & $3.7 \%$ & 28 & $2.6 \%$ & 0.104 \\
\hline \multicolumn{6}{|c|}{ Concomitant medications of interest (pre-period only) } \\
\hline ARB or ACE inhibitor (including combinations) & 952 & $49.0 \%$ & 606 & $55.4 \%$ & $<0.001$ \\
\hline$\beta$-adrenoceptor antagonist & 905 & $46.6 \%$ & 600 & $54.9 \%$ & $<0.001$ \\
\hline Amiodarone & 103 & $5.3 \%$ & 110 & $10.1 \%$ & $<0.001$ \\
\hline Clopidogrel & 173 & $8.9 \%$ & 68 & $6.2 \%$ & 0.009 \\
\hline Statin & 789 & $40.6 \%$ & 452 & $41.4 \%$ & 0.68 \\
\hline Proton-pump inhibitor & 493 & $25.4 \%$ & 295 & $27.0 \%$ & 0.325 \\
\hline $\mathrm{H}_{2}$-receptor antagonist & 85 & $4.4 \%$ & 44 & $4.0 \%$ & 0.649 \\
\hline Warfarin & 713 & $36.7 \%$ & 881 & $80.6 \%$ & $<0.001$ \\
\hline \multicolumn{6}{|l|}{ Utilization and expenditures (pre-period only) } \\
\hline Inpatient hospitalization & 207 & $10.6 \%$ & 145 & $13.3 \%$ & 0.031 \\
\hline Medical costs, \$US & 8239 & 19464 & 9754 & 20710 & 0.044 \\
\hline
\end{tabular}

\footnotetext{
a Bridged=inpatient warfarin with inpatient LMWH/PS and/or with UFH.
}

b Non-bridged = inpatient warfarin without inpatient LMWH/PS or UFH.

$\mathbf{A C E}=$ angiotensin converting enzyme; $\mathbf{A R B}=$ angiotensin II type 1 receptor antagonist (angiotensin receptor blocker); $\mathbf{C H A}_{2} \mathbf{D S} \mathbf{S}_{2}-\mathbf{V A S c} \mathbf{s c o r e}=(\mathrm{i})$ Congestive heart failure or left ventricular dysfunction, 1 pt, (ii) Hypertension, 1 pt, (iii) Age $\geq 75$ years, 2 pts, (iv) Diabetes mellitus, 1 pt, (v) Stroke, transient ischemic attack, or thromboembolism, 2 pts, (vi) Vascular disease (prior myocardial infarction, peripheral artery disease, aortic plaque), 1 pt, (vii) 65 $\leq$ Age $<75,1$ pt, (viii) Sex category, 1 pt if female; $\mathbf{C O P D}=$ chronic obstructive pulmonary disease; GERD=gastroesophageal reflux disease; $\mathbf{L M W H} / \mathbf{P S}=$ low molecular weight heparin/pentasaccharide; $\mathbf{N}=$ number of patients in database sample meeting study selection criteria; $\mathbf{n}=$ number of patients in database sample meeting study selection criteria with specified demographic characteristic; NVAF = non-valvular atrial fibrillation; TIA =transient ischemic attack; UFH= unfractionated heparin; VTE=venous thromboembolism. 


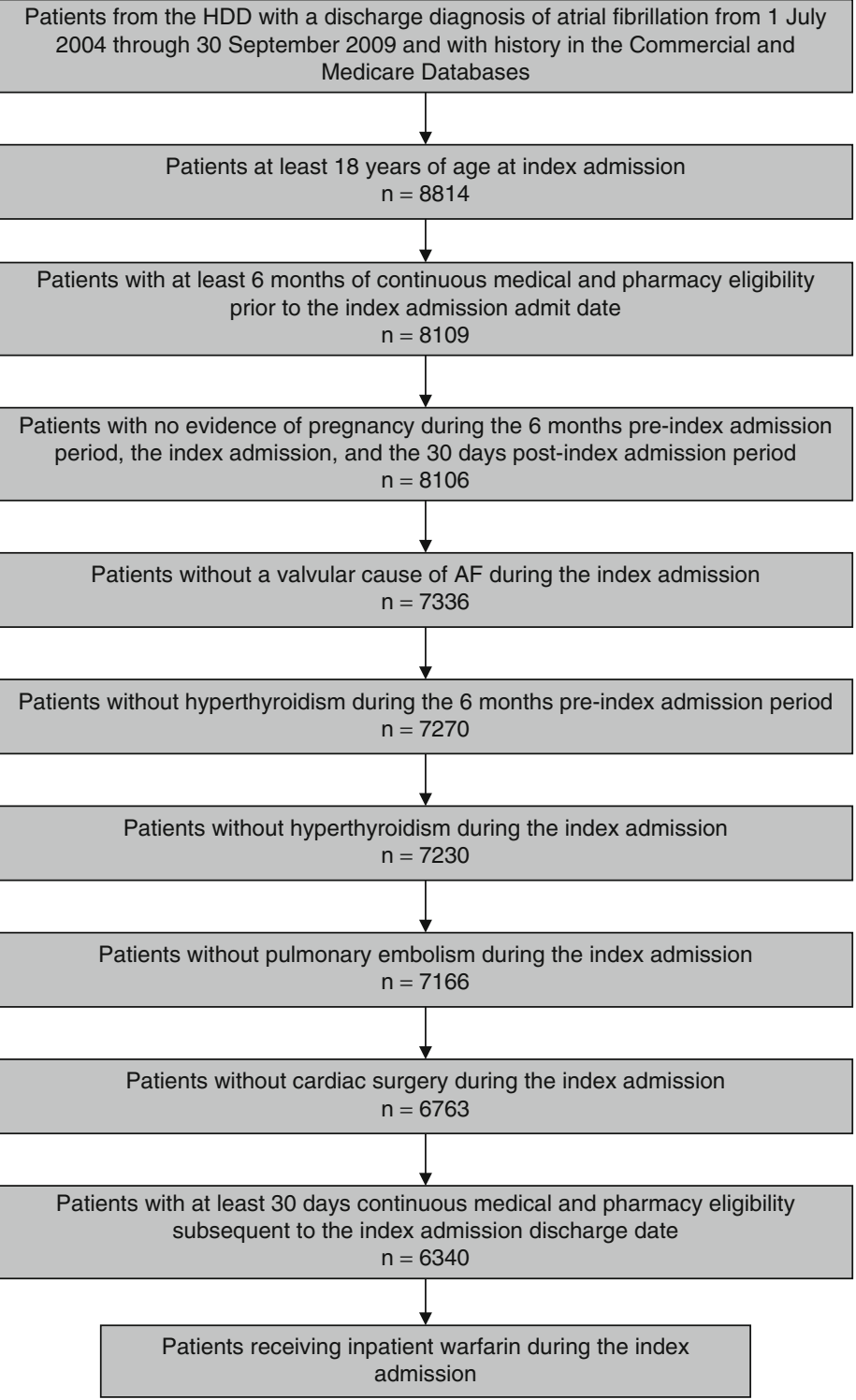

Fig. 1. Study attrition table of NVAF patients. $\mathbf{A F}=$ atrial fibrillation; $\mathbf{H D D}=$ Hospital Drug Database; NVAF = non-valvular atrial fibrillation.

Among bridged patients, only $36.7 \%$ had received warfarin in the 6 months prior to the index admission, compared with $80.6 \%$ of non-bridged patients $(\mathrm{p}<0.001)$ [table II]. Bridged patients were more likely to have a primary discharge diagnosis of AF than non-bridged patients $(37.9 \%$ vs $20.8 \%, \mathrm{p}<0.001)$ [table III]. Although inpatient mortality was low regardless of bridging status, death occurred in $14(0.7 \%)$ of the 1944 bridged patients versus none of the 1093 non-bridged patients $(\mathrm{p}=0.005)$.

Overall, LOS was longer in bridged patients (mean 6.3, SD 5.9, median 5 days) than in those receiving warfarin alone (mean 4.2, SD 3.1, median 3 days). Patients receiving LMWH/ PS as the bridging agent had the shortest LOS (mean 5.6, SD
5.3, median 4 days) of the bridging regimens, while those receiving two-agent bridging had the longest LOS (mean 8.4, SD 8.4 , median 6 days).

\section{Multivariate Analysis}

Multivariate analysis estimated that compared with patients who were on warfarin only, patients with a bridging regimen had an $18.2 \%$ higher LOS $(p=0.0017$, data not shown). Multivariate analysis was also used to investigate the change in LOS associated with each of the bridging regimens, controlling for demographic and clinical characteristics. Results are presented for the change in one variable from the reference population, with the exception of the bridge agent interactions, which report the change in LOS for two variables compared with the reference (table IV). The predicted LOS for the warfarin-only reference group was 3.74 days. Compared with the reference LOS, patients receiving inpatient warfarin and $\mathrm{UFH}$ had a $19.3 \%$ increase in $\operatorname{LOS}(\mathrm{p}=0.0030)$, while those receiving inpatient warfarin and two-agent bridging had a $45.1 \%$ increase in $\operatorname{LOS}(\mathrm{p}<0.0001)$. Although patients bridged with LMWH/PS had an increase in LOS of $8.3 \%$, it was not significant $(\mathrm{p}=0.1577)$. To put the differences in LOS among bridged patients in perspective, LOS was reported for non-bridged patients in comparison with the non-bridged reference patient for selected conditions. Female patients had a $6 \%$ shorter LOS than the reference male patient $(p=0.0133)$. Every increase of 1 year in age above 73 years resulted in a $1.2 \%$ increase in LOS $(\mathrm{p}<0.0001)$. Patients receiving warfarin in the pre-period had an $8.5 \%$ shorter LOS than the reference patient who received warfarin during the inpatient stay only, although the difference was not significant at the 0.05 level $(p=0.0814)$.

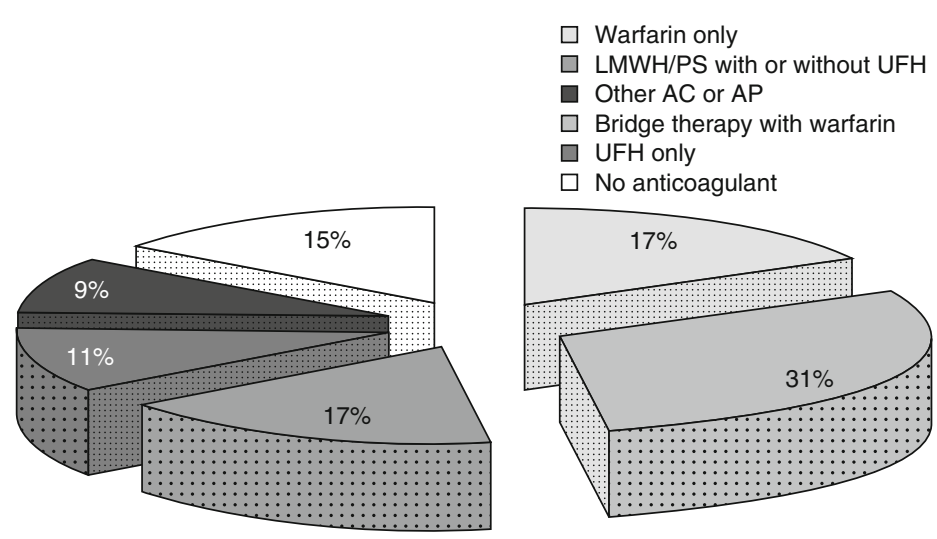

Fig. 2. Anticoagulant $(A C)$ /antiplatelet $(A P)$ use for NVAF patients during index hospitalization $(n=6340)$. LMWH/PS = low molecular weight heparin/ pentasaccharide; NVAF = non-valvular atrial fibrillation; $\mathbf{U F H}=$ unfractionated heparin. 
Table III. Index admission status and drug use

\begin{tabular}{|c|c|c|c|c|c|}
\hline \multirow[t]{3}{*}{ Status or drug use } & \multicolumn{4}{|c|}{ NVAF patients on warfarin } & \multirow[t]{3}{*}{$\mathrm{p}$-Value } \\
\hline & \multicolumn{2}{|c|}{ Bridged $^{a}(\mathrm{~N}=1944)$} & \multicolumn{2}{|c|}{ Non-bridged $^{\mathrm{b}}(\mathrm{N}=1093)$} & \\
\hline & $\mathrm{n}$ & $\%$ & $\mathrm{n}$ & $\%$ & \\
\hline In-hospital mortality & 14 & 0.7 & 0 & 0 & 0.005 \\
\hline AF principal discharge diagnosis & 737 & 37.9 & 227 & 20.8 & $<0.001$ \\
\hline Admitted from emergency room & 1113 & 57.3 & 604 & 55.3 & 0.288 \\
\hline \multicolumn{6}{|l|}{ Inpatient anticoagulant use } \\
\hline Warfarin & 1944 & 100.0 & 1093 & 100.0 & $\mathrm{n} / \mathrm{a}$ \\
\hline LMWH & 1226 & 63.1 & 0 & 0.0 & $<0.001$ \\
\hline UFH & 1062 & 54.6 & 0 & 0.0 & $<0.001$ \\
\hline Fondaparinux (PS) & 15 & 0.8 & 0 & 0.0 & 0.004 \\
\hline Argatroban & 1 & 0.1 & 1 & 0.1 & 0.68 \\
\hline Bivalirudin & 24 & 1.2 & 4 & 0.4 & 0.016 \\
\hline Lepirudin & 0 & 0.0 & 0 & 0.0 & $\mathrm{n} / \mathrm{a}$ \\
\hline Aspirin (acetylsalicylic acid) & 829 & 42.6 & 265 & 24.2 & $<0.001$ \\
\hline Clopidogrel & 207 & 10.6 & 62 & 5.7 & $<0.001$ \\
\hline Aspirin + clopidogrel & 136 & 7.0 & 29 & 2.7 & $<0.001$ \\
\hline Rate control medications & 1805 & 92.8 & 943 & 86.3 & $<0.001$ \\
\hline Antiarrhythmic medications & 1142 & 58.7 & 486 & 44.5 & $<0.001$ \\
\hline
\end{tabular}

a Bridged = inpatient warfarin with inpatient LMWH/PS and/or with UFH.

b Non-bridged = inpatient warfarin without inpatient LMWH/PS or UFH.

$\mathbf{A F}=$ atrial fibrillation; $\mathbf{L M W H}=$ low molecular weight heparin; $\mathbf{L M W H} / \mathbf{P S}=\mathrm{LMWH} /$ pentasaccharide; $\mathbf{N}=$ number of patients in database sample meeting study selection criteria; $\mathbf{n}=$ number of patients in database sample meeting study selection criteria with specified demographic characteristic; $\mathbf{n} / \mathbf{a}=$ not applicable; $\mathbf{N V A F}=$ non-valvular atrial fibrillation; $\mathbf{P S}=$ pentasaccharide; $\mathbf{U F H}=$ unfractionated heparin.

There were no significant changes in predicted LOS from the reference population for individual variables of pre-period warfarin use or occurrence of cancer or diabetes. Thus among NVAF patients not receiving bridging therapy, there was no difference in LOS for those with cancer versus those without cancer. However, significant increases in predicted LOS were observed for NVAF patients with cancer who received bridging therapy compared with the reference NVAF group without cancer and without bridging therapy. Similarly, among nonbridged groups, the models did not predict differences in LOS for NVAF patients with pre-period warfarin use or diabetes as compared with the reference NVAF group. As with cancer, estimated LOS was higher for patients with pre-period warfarin use or with diabetes in conjunction with inpatient bridging therapy. The greatest increase in $\operatorname{LOS}(101.2 \%, \mathrm{p}=0.0107)$ was found for patients with cancer who received two-agent (LMWH/ PS and UFH) bridging with inpatient warfarin. Predicted LOS for these patients was 7.53 days, compared with the reference LOS of 3.74 days (no cancer and no bridging therapy) and the predicted LOS for cancer and no bridging therapy of 4.05 days (figure 3). Similarly, patients with diabetes who also received bridging had a $24.5 \%$ increase in LOS with LMWH/PS, $21.1 \%$ higher with UFH, and $54.4 \%$ higher with both bridging agents than the reference LOS of 3.74 days for a non-diabetic AF patient receiving inpatient warfarin (table IV). For the three clinical variables shown here, no differences were predicted in LOS as compared with the reference group, but the addition of bridging therapy resulted in longer predicted LOS for each variable.

\section{Discussion}

The current study found that less than half of NVAF patients received warfarin while hospitalized. Among patients receiving inpatient warfarin, nearly two-thirds also received inpatient bridging therapy. LMWH/PS was the most common bridging agent, followed by UFH. Only $18 \%$ of patients with bridging therapy received both LMWH/PS and UFH. Patients receiving bridging therapy were younger and generally in better health, with slightly lower $\mathrm{CCI}$ and $\mathrm{CHA}_{2} \mathrm{DS}_{2}$-VASc scores, 
lower rates of previous hospitalization, and lower pre-period healthcare costs. Bridged patients had higher rates of atrial flutter, ischemic stroke/TIA/cerebrovascular disease, and acute myocardial infarction (AMI), conditions associated with higher thrombotic risk, but only $36.7 \%$ of bridged patients had evidence of warfarin use in the 6 months before hospitalization. The study used observational data to describe rather than predict receipt of bridging therapy and thus the reasons for bridging are unknown. However, the results suggest that patients without

Table IV. Predicted change in length of stay

\begin{tabular}{|c|c|c|}
\hline \multirow[t]{2}{*}{ Variables } & \multicolumn{2}{|c|}{$\begin{array}{l}\text { NVAF Patients treated with warfarin } \\
(\mathrm{N}=3037)\end{array}$} \\
\hline & $\begin{array}{l}\% \text { Change in length of } \\
\text { stay relative to reference }\end{array}$ & $\mathrm{p}$-Value \\
\hline Bridge: LMWH/PS & 8.3 & 0.1577 \\
\hline Bridge: UFH & 19.3 & 0.0030 \\
\hline Bridge: two-agent & 45.1 & $<0.0001$ \\
\hline Age above 73 years $^{b}$ & 1.2 & $<0.0001$ \\
\hline Female & -6.0 & 0.0133 \\
\hline Pre-period warfarin & -8.5 & 0.0814 \\
\hline $\begin{array}{l}\text { Bridge therapy: LMWH/PS and } \\
\text { pre-period warfarin }^{c}\end{array}$ & 20.8 & 0.0047 \\
\hline $\begin{array}{l}\text { Bridge therapy: UFH and pre-period } \\
\text { warfarin }^{c}\end{array}$ & 21.7 & 0.0066 \\
\hline $\begin{array}{l}\text { Bridge therapy: two-agent and } \\
\text { pre-period warfarin }^{c}\end{array}$ & 30.6 & 0.0089 \\
\hline Cancer & 8.3 & 0.2941 \\
\hline $\begin{array}{l}\text { Bridge therapy: LMWH/PS and } \\
\text { cancer }^{c}\end{array}$ & 36.7 & 0.0148 \\
\hline Bridge therapy: UFH and cancer ${ }^{\mathrm{C}}$ & 55.2 & 0.0013 \\
\hline $\begin{array}{l}\text { Bridge therapy: two-agent and } \\
\text { cancerc }^{c}\end{array}$ & 101.2 & 0.0107 \\
\hline Diabetes mellitus & 1.1 & 0.8017 \\
\hline $\begin{array}{l}\text { Bridge therapy: LMWH/PS and } \\
\text { diabetes }^{c}\end{array}$ & 24.5 & 0.0025 \\
\hline Bridge therapy: UFH and diabetes ${ }^{c}$ & 21.1 & 0.0237 \\
\hline $\begin{array}{l}\text { Bridge therapy: two-agent and } \\
\text { diabetes }^{c}\end{array}$ & 54.4 & 0.0004 \\
\hline \multicolumn{3}{|c|}{ Payments of 8.8 (thousands of \$US), and all other variables 'No.' } \\
\hline \multicolumn{3}{|c|}{$\begin{array}{l}\text { For the continuous variable Age, the percentage change is for an increase } \\
\text { of } 1 \text { year. }\end{array}$} \\
\hline \multicolumn{3}{|c|}{$\begin{array}{l}\text { c The percentage change for the interactions reflects the value of the } \\
\text { synergistic effects above those of bridge therapy and pre-period warfarin, }\end{array}$} \\
\hline
\end{tabular}

LMWH/PS = low molecular weight heparin/pentasaccharide; LOS = length of stay; $\mathbf{N V A F}=$ non-valvular atrial fibrillation; $\mathbf{U F H}=$ unfractionated heparin.

Adis $\odot 2012$ Springer International Publishing AG. All rights reserved. evidence of warfarin in the pre-period who received bridging would likely have received rapid onset parenteral agents along with initiation of warfarin, for long-term use. The higher rates among the bridged group of patients with AF as a principal discharge diagnosis, as well with evidence of AMI, stroke, and atrial flutter, also suggest that the majority of the observed bridging was for rapidly establishing effective AC by using a parenteral agent in association with initiation of warfarin. Subsequent research, incorporating clinical data in addition to that available in administrative claims, is needed to understand clinical decision-making regarding inpatient bridging in NVAF patients.

A longer LOS was found in patients bridged during hospitalization (mean 6.3 days, median 5 days) compared with the LOS for patients receiving inpatient warfarin alone (mean 4.2 days, median 3 days). Significant increases in LOS remained for UFH and two-agent bridging after adjustment for demographic and clinical variables including diabetes and cancer. While the NVAF patients in the current study were not limited to those with surgical indications for bridging, the LOS results are nevertheless consistent with studies of patients with periprocedural bridging. Tolosana et al. ${ }^{[21]}$ found a median of 5 days for bridged versus 2 days for non-bridged patients at high risk for thromboembolism and requiring implant or replacement of a pacemaker or implantable cardiac defibrillator. As in the current study, where patients receiving LMWH/PS alone as the bridging agent had the shortest LOS compared with either UFH alone or UFH with LMWH/PS, Spyropoulos et al. ${ }^{[22]}$ found that LOS was decreased in patients bridged in the hospital with LMWH versus UFH (4.6 vs 10.3 days). Of the 901 patients requiring bridging for elective surgery or procedures in Spyropoulos et al., ${ }^{[22]} 74.5 \%$ were receiving oral AC for arterial indications, while $25.5 \%$ had venous indications. AF (without mechanical heart valves) accounted for $38.7 \%$ of the 901 patients. The ACCP recommends SC LMWH over UFH for bridging therapy, ${ }^{[23]}$ and bridging with UFH requires longer LOS than bridging with SC LMWH, which, unlike IV UFH, can be home-administered. ${ }^{[24]}$

Given that in the current study, bridged patients were somewhat younger and healthier than non-bridged patients, it would not have been unreasonable to expect a shorter LOS in these patients if bridging had no effect on LOS. Yet, following multivariate analysis, LOS was significantly longer for patients bridged with UFH or two-agent bridging. Also, although the rate $(0.7 \%)$ and number of inpatient deaths was small, all 14 deaths were among patients receiving bridging therapy. This finding is contrary to Billet et al. ${ }^{[15]}$ who found model-adjusted mortality rates to be lower among bridged patients, and thus 


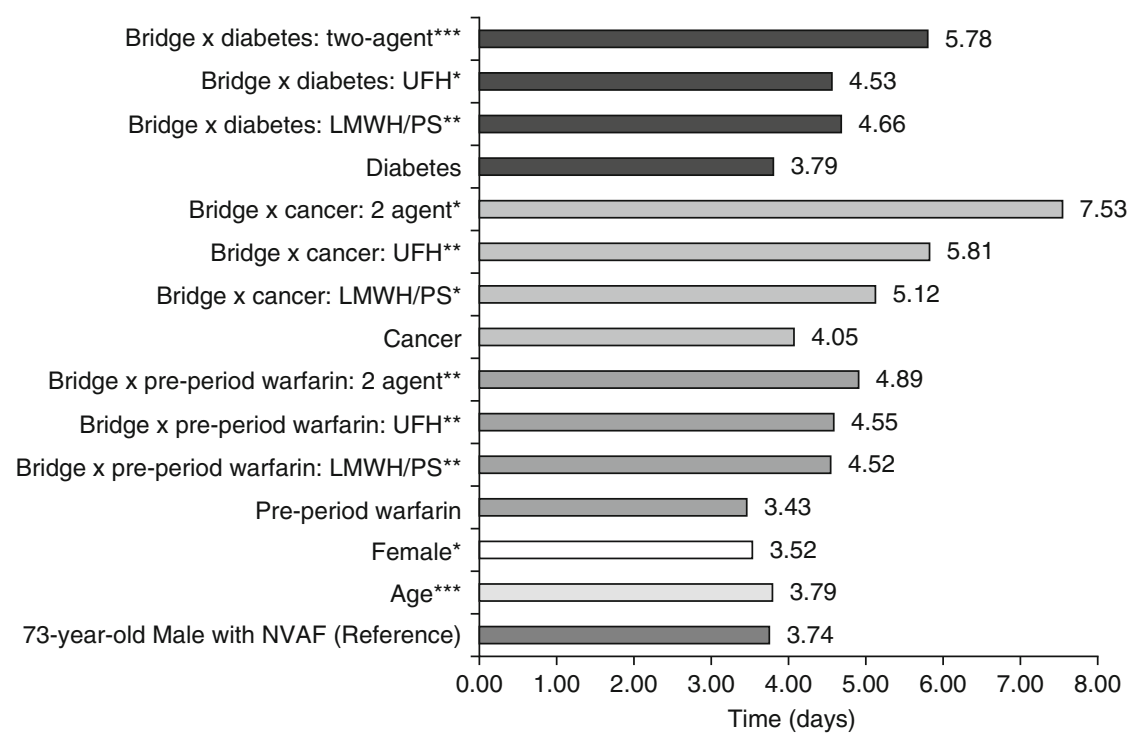

Fig. 3. Estimated LOS during Index hospitalization by bridging regimen and selected variables. The reference LOS is 3.74 days for male aged 73 years, not from the Western region, with total 6-month pre-period costs of $\$$ US8800 and all other variables equal to 'No'. Calculation of LOS reported for interaction terms (e.g. bridge $\times$ cancer: LMWH/PS) includes main effects and interaction effects. LOS=length of stay; LMWH/PS=low molecular weight heparin/ pentasaccharide; NVAF $=$ non-valvular atrial fibrillation; UFH $=$ unfractionated heparin. ${ }^{*} p<0.05 ;{ }^{* *} p<0.01 ;{ }^{* * *} p<0.001$ vs reference.

our mortality results may not be robust to adjustment. The discrepancy in mortality results, as well as the increased LOS among bridged patients, suggests that the benefits versus risks of bridging therapy, as well as possible alternatives, are worth further assessment.

Due to the limitations of observational data, the reason for longer LOS in NVAF patients receiving inpatient bridging could not be ascertained. One hypothesis is that the bridging procedure itself may delay an invasive procedure or discharge until therapeutic levels of AC are established. An alternate hypothesis is that bridging could be a marker of patient complexity not evident in the co-morbidity indicators investigated here, and thus both the decision to bridge and the observed longer LOS may be caused by unobservable factors requiring closer monitoring or additional care. Subsequent research is needed that is designed to identify and evaluate both the causes for bridging and for the longer LOS observed in patients receiving bridging therapy.

Unexpected findings of this study were that, although patients bridged with LMWH/PS did not have statistically significant longer stays than a similar reference group, those with diabetes or cancer who were bridged with LMWH/PS (as well as with UFH or both agents) had longer LOS than the nonbridged reference patients without these conditions. However, non-bridged patients with cancer or with diabetes did not have longer LOS than the non-bridged reference group without these conditions. Bridging is associated with longer LOS for a range of co-morbid conditions and may interact with selected con- ditions, resulting in longer LOS than for bridged patients without these conditions. Since both cancer and diabetes are complex, it is possible that the bridged group with these conditions had a higher level of severity, unobservable in claims data, which may have resulted in both longer LOS and a higher likelihood to receive bridging therapy. The reasons for this finding cannot be assessed from our study design but are intriguing questions for subsequent analyses. Previous research has suggested that a range of co-morbidities, including certain types of cancer, may affect the expected therapeutic effect of warfarin and that AC may confer survival advantages to patients with solid tumors. ${ }^{[1]}$

This study adds to the existing evidence that bridging therapy results in an increased LOS compared with inpatient receipt of warfarin alone, and that these increases in LOS associated with bridging are greater for patients with a range of comorbidities such as cancer and diabetes. Given this outcome, it is clearly important to weigh the clinical benefits against the risks of bridging as a means of managing $\mathrm{AC}$ in the NVAF patient during hospitalization. While the average risk of stroke in NVAF patients not treated with $\mathrm{AC}$ is reported to be $4.5 \%,{ }^{[22]}$ risk varies considerably depending on age and selected comorbidities such as diabetes, congestive heart failure, hypertension, and prior stroke or TIA. ${ }^{[25,26]}$ Determining when the risk of thromboembolism is sufficiently high to require bridging during warfarin initiation, during periods of subtherapeutic AC in patients already treated with warfarin, or during interruption of warfarin peri-operatively, is challenging. Two recent reviews, 
focused on patients undergoing surgery or procedures, have attempted to sort through the available data and to provide guidance to physicians faced with this decision. ${ }^{[25,26]}$ Kaatz and Paje $^{[25]}$ cite a 30-day risk of stroke in AF patients with discontinuation of $\mathrm{AC}$ and without bridging of $0.6-1.8 \%$, increasing to greater than $3 \%$ in patients undergoing more complex surgery. The $\mathrm{CHA}_{2} \mathrm{DS}_{2}$-VASc score provides a means for assessing thromboembolic risk. ${ }^{[20]}$ Various approaches have been used, including type and length of the surgical procedure, the significance of the potential bleed, and subjective determination by the physician. ${ }^{[25,26]}$ The HAS-BLED bleeding risk score, which incorporates diagnostic as well as laboratory data such as INR values shows promise, ${ }^{[27]}$ although HAS-BLED cannot readily be applied in studies such as ours, which use administrative claims data. Future research should include factors such as bleeding and thrombosis to better understand clinical decision-making regarding bridging therapy and why it results in longer LOS.

Several limitations to the study should be noted. First, this was a retrospective, observational study based on administrative claims data and therefore the reasons for patient receipt of inpatient bridging or explanation for longer LOS among bridged than non-bridged patients could not be determined. Another limitation of this type of study is that the selection of patients with NVAF is limited by completeness and accuracy of medical coding. However, as selection was based on hospitalization discharge diagnosis codes, concern over inclusion of patients based on rule-out diagnoses in the outpatient setting or admission diagnoses in the inpatient setting would not be an issue. Third, so as to provide a broad view of bridging therapy in the inpatient setting, medical and surgical admissions were not differentiated. Fourth, factors impacting LOS were identified from demographic and clinical variables that could be defined using administrative claims data. The relation to LOS for variables not captured in these data but which might have influenced LOS, such as bleeding risk as identified by HAS-BLED scoring, cannot be determined from this study. Fifth, differences in LOS from the reference group were shown for one variable at a time and for the interaction of bridging agents with pre-period warfarin, cancer, and diabetes, as well as other co-morbidities not reported here. Interactions with other drugs and inpatient procedures were not evaluated, and LOS was not evaluated for multiple combinations of demographic or clinical conditions different to the reference population. Sixth, the current study defined bridging as inpatient receipt of warfarin in addition to LMWH/PS, UFH, or two-agent bridging, without regard to the timing of the agents. Thus bridging that occurred in the outpatient setting, including peri-operative bridging pre-admission or post-discharge, was not captured. In addition, LMWH use prior to the index admission and procedures performed during the index hospitalization were not tracked, and, therefore, their impact on observed LOS could not be determined. Finally, the study population was comprised of commercially insured patients and patients with employer-paid supplemental Medicare coverage, and results may not be representative of all patients with NVAF, especially the uninsured or those covered by Medicaid.

\section{Conclusion}

This retrospective observational study found that, in patients hospitalized with NVAF, inpatient bridging therapy was common among those under warfarin therapy, and more common in patients who had no previous warfarin use and had thrombotic risk. Bridging therapy was also found to be associated with a longer LOS. Patients receiving inpatient warfarin only had shorter LOS than those receiving warfarin in conjunction with inpatient LWMH/PS, UFH, or both types of bridging agents. Additional research is needed to identify predictors of inpatient bridging therapy as well as to explain why, even after controlling for demographic and clinical characteristics, NVAF patients receiving inpatient bridging therapy had longer LOS than those who were not bridged.

\section{Acknowledgments}

This study and preparation of the manuscript was funded by Boehringer Ingelheim Pharmaceuticals, Inc. (BIPI). KST, BHJ, and DMS are employees of Truven Health Analytics Inc., which was contracted by BIPI to work in collaboration on this study. KS, DWW, and SS are employees of BIPI. AA, of University of California, Irvine, was a paid consultant hired by BIPI for expertise on this topic. KST, BHJ, KS, DRW, SS, and AA contributed to study concept and design, and to data interpretation. BHJ and DMS performed the analyses. KST led the writing of the manuscript, and all authors critically reviewed it and gave their final approval of the version to be published.

All authors meet criteria for authorship as recommended by the International Committee of Medical Journal Editors (ICMJE), were fully responsible for all content and editorial decisions, and were involved at all stages of manuscript development. The authors wish to acknowledge Daniel Huse of Thomson Reuters for his clinical and methodological expertise, and the programming assistance of Suman Jannuru and Boris Ivanov, also of Truven Health Analytics Inc., in addition to Michele Shaw of Naples, FL, USA for her medical writing expertise.

\section{References}

1. Kim MH, Johnston SS, Chu BC, et al. Estimation of total incremental health care costs in patients with atrial fibrillation in the United States. Circ Cardiovasc Qual Outcomes 2011; 4: 313-20 
2. Wu EQ, Birnbaum HG, Mareva M, et al. Economic burden and co-morbidities of atrial fibrillation in a privately insured population. Curr Med Res Opin 2005; $21: 1693-9$

3. Mercaldi CJ, Ciarametaro M, Hahn B, et al. Cost efficiency of anticoagulation with warfarin to prevent stroke in Medicare beneficiaries with nonvalvular atrial fibrillation. Stroke 2011; 42: 112-8

4. Caro JJ. An economic model of stroke in atrial fibrillation: the cost of suboptimal oral anticoagulation. Am J Manag Care 2004; 10: S451-61

5. Naccarelli GV, Varker H, Lin J, et al. Increasing prevalence of atrial fibrillation and flutter in the United States. Am J Cardiol 2009; 104: 1534-9

6. Kannel WB, Wolf PA, Benjamin EJ, et al. Prevalence, incidence, prognosis, and predisposing conditions for atrial fibrillation: population-based estimates. Am J Cardiol 1998; 82: 2N-9N

7. Tsivgoulis G, Spengos K, Zakopoulos N, et al. Efficacy of anticoagulation for secondary stroke prevention in older people with non-valvular atrial fibrillation: a prospective case series study. Age Ageing 2005; 34: 35-40

8. Lloyd-Jones D, Adams RJ, Brown TM, et al. Heart disease and stroke statistics - 2010 update: a report from the American Heart Association. Circulation 2010; 121:e46-e215

9. Goldstein LB, Bushnell CD, Adams RJ, et al. Guidelines for the primary prevention of stroke: a guideline for healthcare professionals from the American Heart Association/American Stroke Association. Stroke 2011; 42: 517-84

10. Furie KL, Kasner SE, Adams RJ, et al. Guidelines for the prevention of stroke in patients with stroke or transient ischemic attack: a guideline for healthcare professionals from the American Heart Association/American Stroke Association. Stroke 2011; 42: 227-76

11. Singer DE, Albers GW, Dalen JEA, et al. Antithrombotic therapy in atrial fibrillation: American College of Chest Physicians evidence-based clinical practice guidelines (8th edition). Chest 2008; 133: 546S-92S

12. Du Breuil AL, Umland EM. Outpatient management of anticoagulation therapy. Am Fam Physician 2007; 75: 1031-42

13. Garcia DA, Regan S, Henault LE, et al. Risk of thromboembolism with shortterm interruption of warfarin therapy. Arch Intern Med 2008; 168: 63-9

14. Hallevi H, Albright KC, Martin-Schild S, et al. Anticoagulation after cardioembolic stroke: to bridge or not to bridge? Arch Neurol 2008; 65: 1169-73

15. Billett HH, Scorziello BA, Giannattasio ER, et al. Low molecular weight heparin bridging for atrial fibriallation: is VTE thomboprophylaxis the major benefit? J Thromb Thrombolysis 2010; 30: 479-85

16. Dunn AS, Turpie AG. Perioperative management of patients receiving oral anticoagulants: a systematic review. Arch Intern Med 2003; 163: 901-8
17. Airaksinen KE, Schlitt A, Rubboli A, et al. How to manage antithrombotic treatment during percutaneous coronary interventions in patients receiving long-term oral anticoagulation: to "bridge" or not to "bridge"? Eurointervention 2010; 6: 520-6

18. Ramirez A, Wall TS, Schmidt M, et al. Implantation of cardiac rhythm devices during concomitant anticoagulation or antiplatelet therapy. Expert Rev Cardiovase Ther 2011; 9: 609-14

19. Deyo RA, Cherkin DC, Ciol MA. Adapting a clinical comorbidity index for use with ICD-9-CM administrative databases. J Clin Epidemiol 1992; 45: 613-9

20. Lip GY, Nieuwlaat R, Pisters R, et al. Refining clinical risk stratification for predicting stroke and thromboembolism in atrial fibrillation using a novel risk factor-based approach: the Euro Heart Survey on atrial fibrillation. Chest 2010; 137: 263-72

21. Tolosana JM, Berne P, Mont L, et al. Preparation for pacemaker or implantable cardiac defibrillator implants in patients with high risk of thromboembolic events: oral anticoagulation or bridging with intravenous heparin? A prospective randomized trial. Eur Heart J 2009; 30: 1880-4

22. Spyropoulos AC, Turpie AG, Dunn AS, et al. Clinical outcomes with unfractionated heparin or low-molecular-weight heparin as bridging therapy in patients on long-term oral anticoagulants: the REGIMEN registry. J Thromb Haemost 2006; 4: 1246-52

23. Douketis JD, Berger PB, Dunn AS, et al. The perioperative management of antithrombotic therapy: American College of Chest Physicians evidencebased clinical practice guidelines (8th edition). Chest 2008; 133: 299S-339S

24. Kalafut MA, Gandhi R, Kidwell CS, et al. Safety and cost of low-molecularweight heparin as bridging anticoagulant therapy in subacute cerebral ischema. Stroke 2000; 31: 2563-8

25. Kaatz S, Paje D. Update in bridging anticoagulation. J Thromb Thrombolysis 2011; 31: 259-64

26. Martin MT, Kuchta AM, Nutescu EA. A clinician's guide to perioperative bridging for patients on oral anticoagulation. J Pharm Pract 2010; 23: 303-12

27. Pisters R, Lane DA, Nieuwlaat R, et al. A novel user-friendly score (HASBLED) to assess 1-year risk of major bleeding in patients with atrial fibrillation: the Euro Heart Survey. Chest 2010; 138: 1093-100

Correspondence: Ms Barbara H. Johnson, MBA, Manager, Custom Data Analytics, Truven Health Analytics, Suite 330, 4301 Connecticut Ave, NW, Washington, DC 20008 USA.

E-mail: barbara.h.johnson@truvenhealth.com 